\title{
Design Support for Hypermedia Documents
}

M.D. Apperley and R.B. Hunt

University of Waikato

Hamilton, New Zealand, m.apperley@cs.waikato.ac.nz

\begin{abstract}
The nonlinear nature of hypermedia documents makes them notoriously difficult to describe. Consequently design prior to implementation is a challenging task. This paper examines design issues specific to hypermedia, and describes the development of HANDIE, a notation for the description of the structural organisation of hypermedia documents. HANDIE is based on a directed graph approach, but it incorporates a range of abstractions which provide significant simplification, and which allow the underlying structure of a document to remain clearly visible. The evaluation of a prototype design environment based on HANDIE is described, and range of refinements for the future are proposed.
\end{abstract}

\section{Keywords}

hypermedia, multimedia, design methodology, World-Wide Web, graphical notation 


\section{INTRODUCTION}

Because of their inherent nonlinearity, hypermedia documents are very difficult to describe; in fact, their best description is usually their implementation. As a consequence, attempting to carry out comprehensive organisational and structural design prior to implementation is a challenging task. The majority of hypermedia design aids available are either (i) style guides, relating to appearance and frame-toframe relationships, providing little to support the overall design of the document or system, or (ii) are inextricably tied into a particular formal design methodology. The development and extraordinarily rapid penetration of the World-Wide Web has highlighted the need for tools to assist with WWW site design and maintenance, where the bulk of the issues involved are generic to hypermedia. This need is further exacerbated by the fact that many of those engaged in the design of hypermedia systems and WWW sites have little or no formal background in software systems design (Pohl and Purgathofer, 1994).

This paper examines the critical problems of hypermedia design which set it apart from more conventional software development, and reviews a range of current design tools. From this base, a graphical notation for the description of hypermedia systems is then developed, aimed specifically at supporting the hypermedia design process rather than prescribing a formal design methodology. The prototype implementation of this notation within a hypermedia design environment (HANDIE) is described. Some preliminary feedback from designers who have evaluated the system is also discussed.

\section{HYPERMEDIA DESIGN ISSUES}

Designing hypermedia documents is a complex activity. Nanard and Nanard (1995) identify the need for formal tools to reduce the cognitive load on the document designer, and to reduce the level of complexity in the design process. One approach is to provide the user with abstract semantic types which can be used to describe a complex real-world situation in a relatively simple fashion. For example, EntityRelationship (ER) modelling is used in traditional software design with the provision of abstract types in the form of entities and relationships at the simplest level, followed by more complex semantic constructs such as aggregation and specialisation at higher levels (Chen, 1976). Another approach to reducing complexity is to allow the user to trial portions of the design in an incremental, experimental and evolutionary fashion. This is usually achieved through rapid prototyping where the user is presented with a working prototype of the user interface without the underlying functionality.

This need for formal tools and complexity reduction is not unique to hypermedia design, but rather applies to the design of any software system, and as a consequence the topic has already received considerable attention, with varying 
success. However, as Nanard and Nanard (1995) have pointed out 'An important part of hypertext design concerns aesthetic and cognitive aspects that software engineering environments do not support.'

Hypermedia document design is most commonly carried out or described at the level of the data, rather than at a higher metadata or semantic entity level. Because of the inherent network nature of hypermedia documents, it is tempting to use standard directed graphs for their description. Figure 1 shows a directed graph representation of part of a university WWW site providing information on lecturers and courses. A standard hierarchical structure provides links from an introductory page to an index of lecturers and an index of courses, and from these indexes to individual lecturers and individual courses respectively. However, links are also provided from lecturers to the courses which they teach, and vice versa, and from each node back to the introduction. For just a few lecturers and a few courses, Figure 1 demonstrates the complexity of this directed graph representation. Further, because of the visual complexity of this representation, Figure 1 also shows the apparent opacity of the underlying structure that it represents.

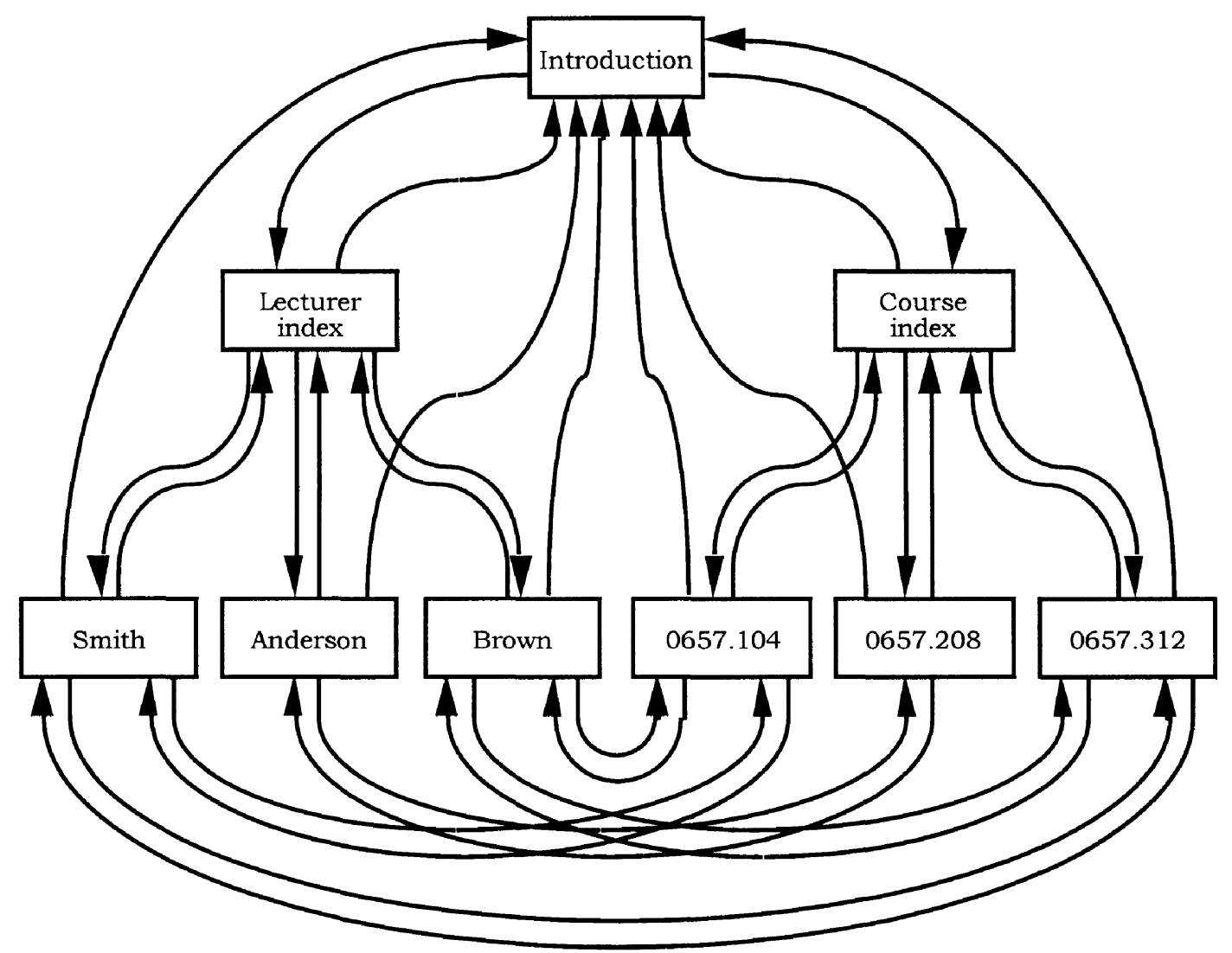

Figure 1 A directed graph representation of a simple hypermedia document, showing the complexity and semantic opacity of representation at this level. 
A further obfuscating aspect of hypermedia documents is that there are potentially three overlapping and conflicting structures, some or all of which may be explicitly represented. These are (i) the logical, inherent or epistemological structure of the information, (ii) the imposed or navigational structure, and (iii) the user's perceived structure (Jul and Furnas, 1997). It is usually only the imposed structure (ii) that is represented in diagrams of the form of Figure 1.

\section{EXISTING DESIGN TOOLS AND METHODOLOGIES}

There are a number of existing tools and procedures that support the hypermedia design process. These variously encompass simple descriptive techniques for the actual structures at the data level, abstraction techniques to allow modelling and description of the conceptual structures, and formal methodologies aimed at improving the design process. The Object Oriented Hypermedia Design Model (OOHDM) (Schwabe and Rossi, 1995; Schwabe et al., 1996) describes hypermedia development as a four-stage process, comprising conceptual design, navigational design, abstract interface design, and implementation, in that sequence. It focuses on navigational and abstract interface design, and encourages re-use. The Relationship Management Methodology (RMM) (Isakowitz et al., 1995) echoes the phases of OOHDM, but adds an additional step between conceptual design and navigational design, that of slice design. Slice design determines how the information defined in the entities (the attributes) of the conceptual model will be grouped and presented to the user, and provides a highlevel partitioning of the logical and imposed structures. For example, for the system of Figure 1 it might be decided to store the following information about each lecturer entity: name, photograph, biography, research area. The slice diagram of Figure 2 shows exactly how this information would be structured in the document, which attributes would be associated with each slice, and how this information could be navigated.

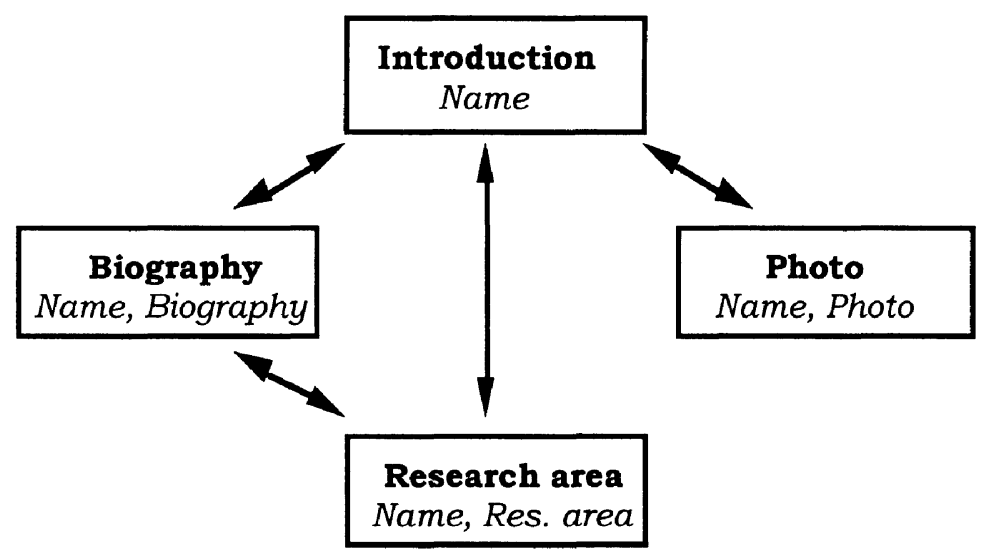

Figure 2 An RMM entity slice diagram. 
The Dexter Hypertext Reference Model (Halasz and Schwartz, 1994) presents a similar approach, and could be regarded as the predecessor of OOHDM and RMM. All three of these methodologies support abstraction and provide an enabling descriptive notation. The Hypertext Analysis, Navigation and Design model (HAND) (Duncan and Apperley, 1994) is simply a visual notation for high-level representation of hypermedia structure, without an accompanying methodology. However, all of these approaches share the common feature that they provide abstract semantic types for specifying structure and navigation, in contrast to earlier design approaches such as that of HyTime, which merely provided mark-up languages for specifying the links between different parts of the document (Newcomb et al., 1991). The semantic types provide by these models range from the simple representation of nodes and links in the Dexter model, to guided tours and entity indexes in RMM, and to classes and groups in HAND.

There are also differences between these models in how the node and link concepts are represented. Some, for example RMM and HAND, regard the node as the atomic unit, and all links are specified as emanating from some source node and terminating at some destination node. Others, notably OOHDM and the Dexter Model, store information about anchors ${ }^{*}$ within node definitions, and thus allow finer-grained link specification, from a source anchor to a destination node. Some models also make a distinction between atomic and composite nodes. Atomic nodes contain a single type of hypermedia, such as a block of text or a video clip, while composite nodes can combine a number of media types in a single node (Duncan and Apperley, 1994).

The discussion so far has dealt specifically with hypermedia. Hypermedia documents increasingly incorporate elements of multimedia as well as hypertext. With applications which include video or sound data, some consideration must be given to timing constraints. The Amsterdam hypermedia model (Hardman et al., 1994) combines multimedia and timing considerations with the abstract node and link representations of the Dexter hypertext reference model. More recently IMMPS (Shih and Davis, 1997) has provided a development environment for multimedia presentations incorporating AI techniques for specifying knowledge inheritance, and a multimedia database.

\section{THE DEVELOPMENT OF A GRAPHICAL NOTATION FOR HYPERMEDIA DOCUMENTS}

The HANDIE hypermedia notation described in this paper, which is based on an extension of the earlier HAND notation (Duncan and Apperley, 1994) has been designed specifically to address those design issues unique and problematic to hypermedia document designers. It makes no attempt to reproduce or replace

See Ginige et al. (1995) for a useful set of definitions of such terms 
methodologies such as RMM or OOHDM, but rather, it aims to provide '...concepts and tools that help produce a design and (sometimes) implement the corresponding product' (Nanard and Nanard, 1995). To this end it must both provide adequate formalisms and at the same time support the incremental and opportunistic activity of the designer (Nanard and Nanard, 1995). These requirements have led to the following four general principles which were adopted in the development of HANDIE:

- Transparency: The notation must be easily understood by users, who may not have formal training in software development.

- Completeness: The model must provide a sufficient range of abstract semantic types to aid the user in reducing the complexity of the hypermedia design process.

- Software Implementability: The model must be able to be supported by a computer-based development environment.

- Continuity of Support: The model should provide for the graceful transition from early design right through to implementation, or at least provide a continuous link in this path.

The HAND notation (Duncan and Apperley, 1994), which provided the basis for HANDIE, already meets a number of these requirements. It concentrates on the structural and navigational phase of design through the use of directed graphs, and incorporates higher level concepts which both simplify the representation of the underlying structure and at the same time make this structure more evident.

The original HAND notation provides four abstract node types, illustrated in Figure 3 (a to d). These node types can be described as follows:

- Basic nodes: These are nodes that represent a single document page. A basic node may contain a single media form, or it may be a composite of several media.

- Group nodes: Group nodes provide a means of representing hierarchy, and simplifying views. A group node corresponds to a section of the document which is represented by a sub-diagram; it can be thought of as 'containing' a collection of nodes and links. A group node is a similar concept to that of an HM s-collection (Maurer et al., 1995) or an RMM slice (Isakowitz et al., 1995).

- Class nodes: A class node represents an homogenous group of related node instances, each with the same underlying structure. Each node instance in the class can be thought of as being similar to a record in a database.

- External nodes: These provide a mechanism for developers to establish links to and from nodes outside the scope of the current document. 
In HANDIE there is one further node type, the list node, illustrated in Figure 3(e):

- List nodes: Class nodes represent homogenous groups of related node instances. A list node provides a means of accessing individual instances of a class node via an index.

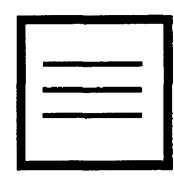

(a)

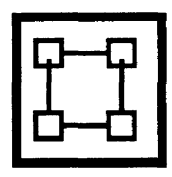

(b)

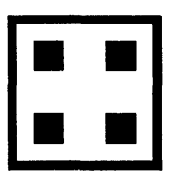

(c)

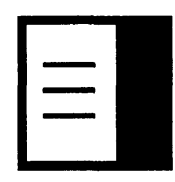

(d)

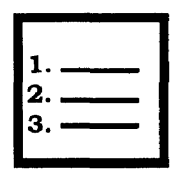

(e)

Figure 3 The five abstract node types of HANDIE, and their representations: (a) The basic node, (b) group node, (c) class node, (d) external node, and (e) a list node.

The HAND notation included two forms of link, simple and complex (Apperley and Duncan, 1994). A simple link represents a direct relationship between two nodes, with a fixed anchor and a fixed destination node. A complex link, however, is a link for which the destination is determined at runtime, on the basis of contextual information. HAND provided no means for specifying this context. HANDIE has expanded and refined the notion of a complex link, to that of a conditional link. A conditional link will always have as its destination a class node, and includes a specification of the condition associated with that link which will determine the actual destination instance at runtime. Examples of conditional links can be seen in Figure 4, where such links are represented by thick lines and are accompanied by associated query specifications.

A further concept that has been included in HANDIE is that of the higraph (Harel, 1988). Higraphs provide for further complexity reduction in directed graph representations by allowing nodes to be grouped into sets; a link specified from a node set to another node is equivalent to a link from every node in that set. The higraph concept as implemented in HANDIE is illustrated in Figure 5. A comparison of the HANDIE representation of Figure 4 and the conventional directed graph of Figure 1, demonstrates how the use of higraphs and class nodes significantly reduces the number of links without compromising the explicitness of a diagram.

To illustrate the use of the group node in HANDIE, consider the situation (from Figure 4) where the lecturer for one course wishes to establish a collection of web pages to support that course. There should be a link to that collection from the course information page, but the detail of this collection is not of part of the design at present. The appropriate representation of this situation, using a group node, is shown in Figure 6. Note here a further use of the conditional link notation. A 
conditional link is used in Figure 6 with a class node (Courses) as its source; in this case it indicates which instance (or set of instances) of the class is the anchor for what is, in effect, a simple link.

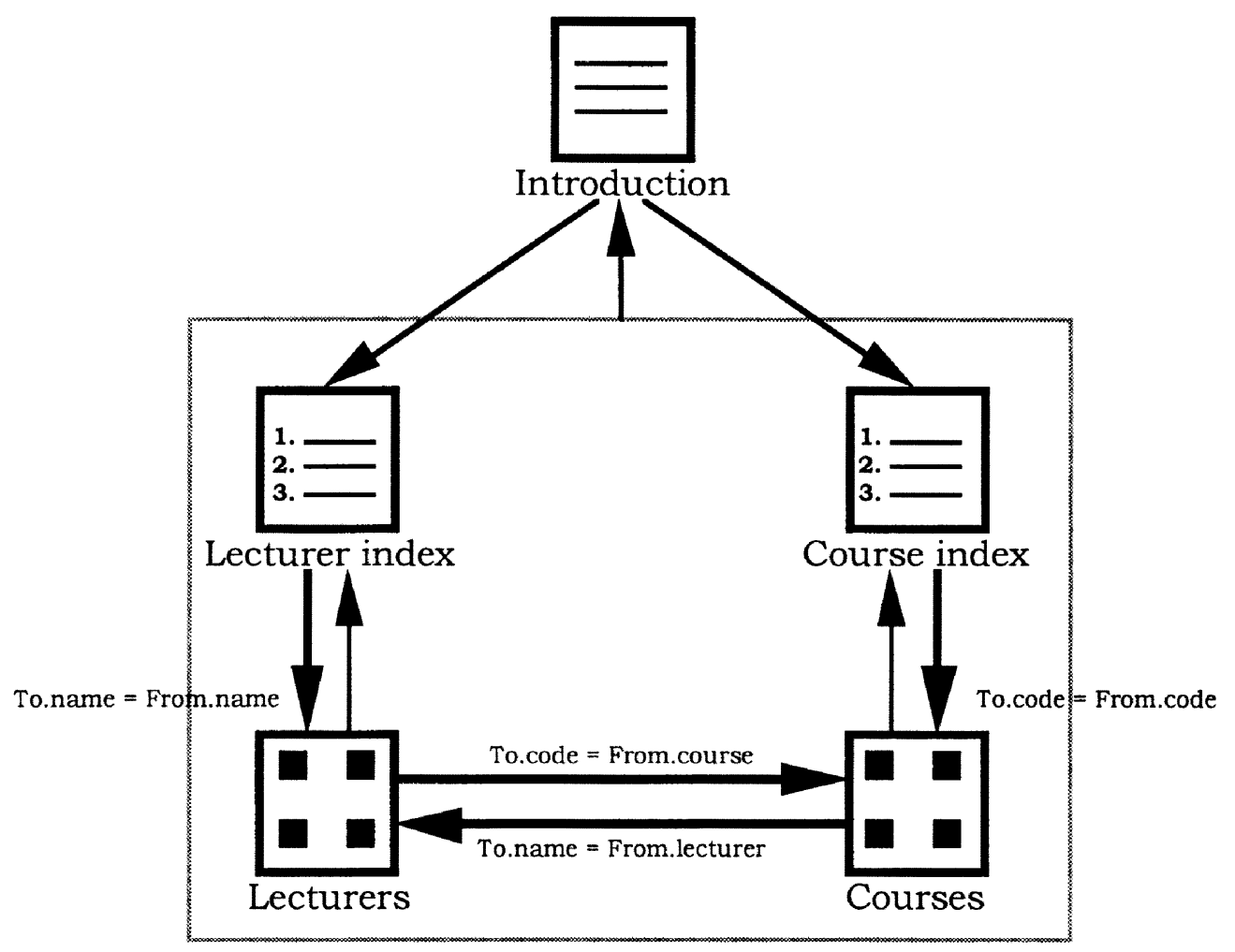

Figure 4 An example document design using HANDIE.

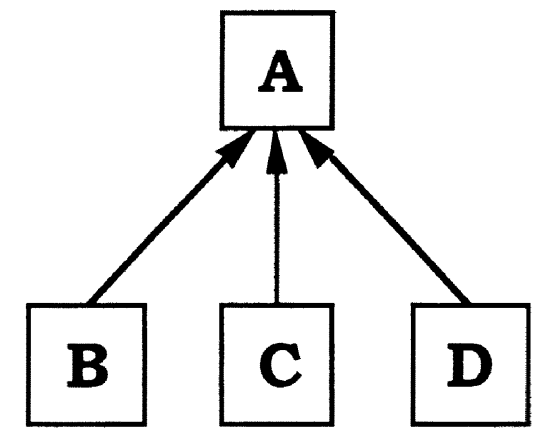

(a)

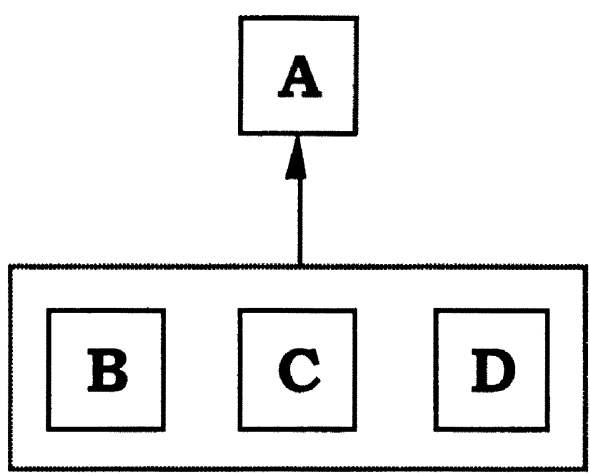

(b)

Figure 5 (a) Standard and (b) higraph enhanced multiple link abstractions.

Figure 6 also provides an illustration of the use of the external node abstraction. This provides a mechanism for a link from the University's home page to the 
introductory node; the home page will always remain outside the scope of the design, yet it is necessary to make reference to it.

HANDIE, as developed at this stage, is concerned principally with the definition of the navigable structure of hypermedia documents, and does not provide full data modelling facilities for specifying the data contained in those documents. Ultimately it is anticipated that modelling tools will be integrated into the HANDIE environment. However, for the present, limited provision has been provided for the specification of existing data models. For each entity, the user is able to specify the relevant attributes. Sets of these entity-attributes can then be associated with individual nodes, and are then available for use in conditions associated with links between nodes. The tables of Figure 7 show the attributes associated with the lecturer and course entities of Figure 6, and the data contents of the index and class nodes. These tables help explain the link conditions in Figure 6.

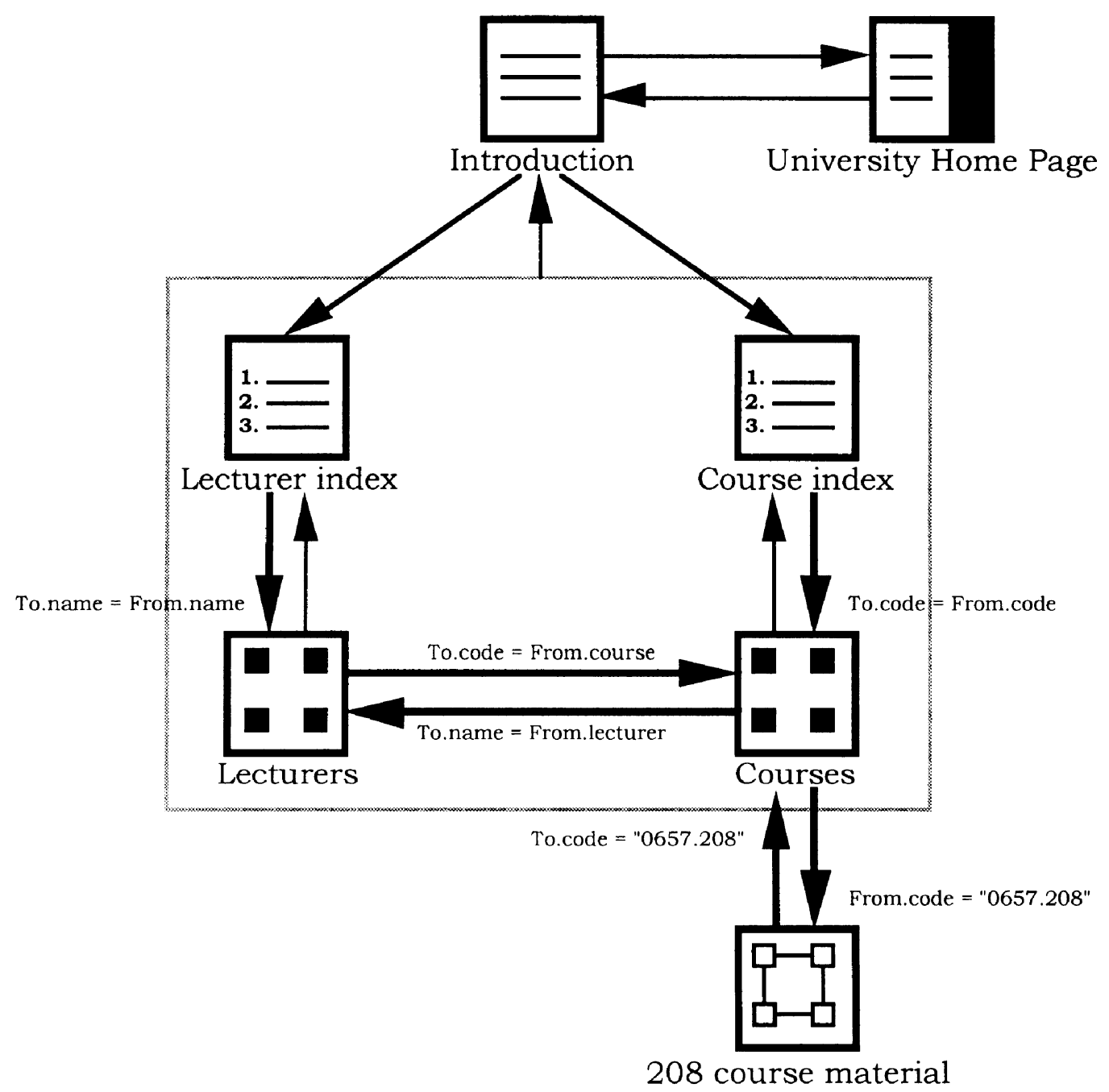

Figure 6 An illustration of the use of the HANDIE group and external node facilities. 


\begin{tabular}{|l|l|}
\hline Entity & Attributes \\
\hline lecturer & $\begin{array}{l}\text { name } \\
\text { photograph } \\
\text { resarea } \\
\text { course }\end{array}$ \\
& $\begin{array}{l}\text { code } \\
\text { lecturer } \\
\text { coom }\end{array}$ \\
\hline
\end{tabular}

(a)

\begin{tabular}{|l|l|}
\hline Node & Data content \\
\hline Lecturer index & lecturer.name \\
Lecturers & $\begin{array}{l}\text { course.code } \\
\text { lecturer.name } \\
\text { lecturer.photo } \\
\text { lecturer.resarea } \\
\text { lecturer.course }\end{array}$ \\
Courses & $\begin{array}{l}\text { course.code } \\
\text { course.lecturer } \\
\text { course.room }\end{array}$ \\
\hline
\end{tabular}

(b)

Figure 7 (a) The data model used in the design of Figure 6, and (b) the data contents of individual nodes.

\section{THE IMPLEMENTATION OF A PROTOTYPE DESIGN ENVIRONMENT}

The HANDIE hypermedia design tool described in the previous section has been implemented as a stand-alone Java application using JDK 1.1.3 in a Windows NT environment. The basic design window of this prototype system is shown in Figure 8. Apart from the appearance of handles on nodes and links, the representation of higraphs, and the display of link conditions, there is little difference between the appearance of the actual design of Figure 8 and that of the idealised form of Figure 6.

Within the HANDIE design window the user is able to specify nodes of specific types from a menu and to drag them to the desired position using the mouse, and to define links by dragging from a handle on the source node to a handle on the destination node. Individual nodes and links can be deleted (via the Node and Link menus) and nodes retain all link connections if they are moved by dragging. The higraph link from the four nodes Lecturer Index, Course Index, Lecturers and Courses is represented by the short sourceless link to Introduction in Figure 8. This is produced by explicitly drawing the four separate links, selecting them as a group, and then selecting Higraph from the Link menu. Clicking on this abbreviated higraph link on the design causes the component links to be displayed in highlighted form. 


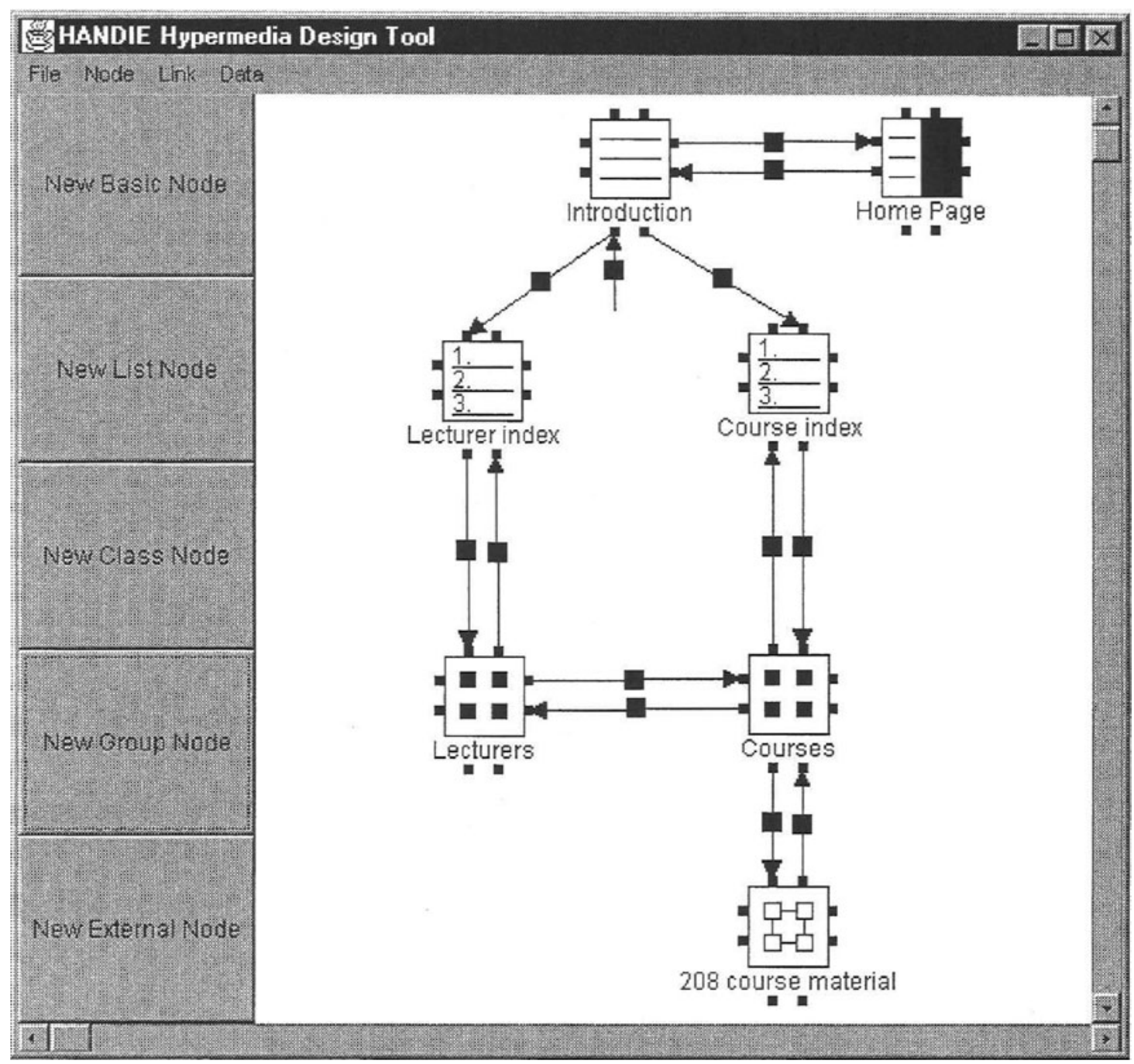

Figure 8 The HANDIE design environment showing the example of Figure 6.

The data model for the design is defined in a separate pop-up window which is accessed through the Data menu in the design window. An example of this data definition window is shown in Figure 9(a). Once this information has been provided, then the data contents for a specific node can be defined and accessed by a double-click on the node (pop-up window of Figure 9b), and conditions associated with a given link can be defined and accessed by a double-click on the link (pop-up window of Figure 9c). Note that for reasons of clarity, link conditions are not permanently displayed as suggested in the idealised form of Figure 6 .

The implementation includes full support for group nodes and hierarchical designs. A double-click on a group node causes a new design window to be opened, within which the lower-level sub-design may be carried out. Through judicious use of this feature, users are able to maintain their designs at a manageable level of complexity and avoid performance limitations that may arise through designs with large numbers of nodes. 


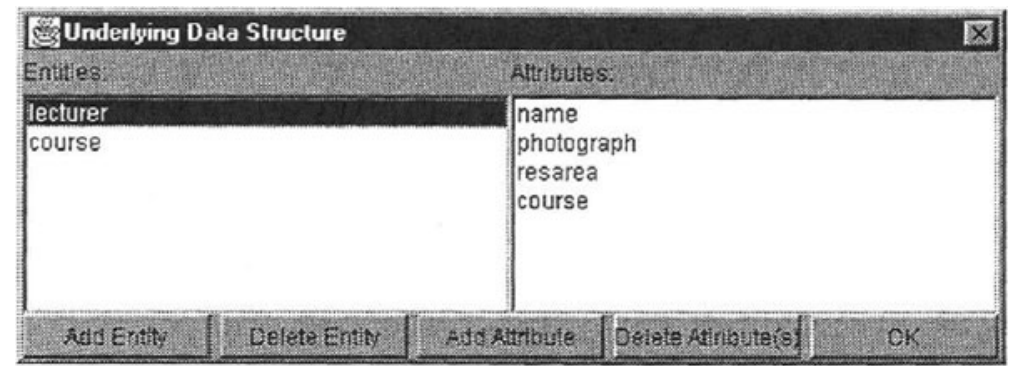

(a)

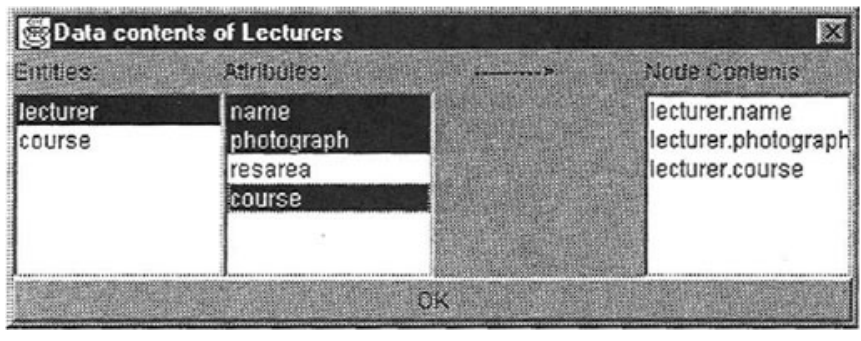

(b)

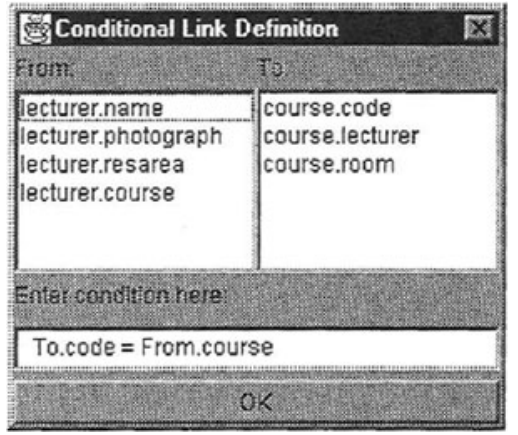

(c)

Figure 9 (a) The data definition pop-up window in which relevant data entities and their attributes are defined, (b) the pop-up window for defining the entityattributes associated with a particular node, and (c) the definition of the condition associated with a link.

\section{PRELIMINARY EVALUATION BY DESIGNERS}

A preliminary evaluation of this prototype tool has been carried out by five users whose work involves hypermedia document design, computer graphic design or WWW page design. In its present prototype form, performance (interactivity) is satisfactory for designs involving no more than about twelve nodes. Users were given a brief introduction to the tool, and then asked to apply it to a design on which they were currently working. After a half-hour session they were asked a series of questions about the usefulness of the HANDIE notation and the tool as implemented. In general, these users found that the notation supported a wide range of hypermedia constructs, and that it was very useful in conceptualising the structure of a document without focusing on its content. In particular, they found the class and list nodes to be very useful abstractions. However, they also found the notation limiting, with two constructs in particular noted as absent: 
- There is no provision for representing a node containing more than one list.

- Class nodes of lists, which would allow lists to point to lists (hierarchical menus) are not supported.

These designers also felt that although the group node abstraction provided a useful partitioning of a design, inevitably designers would want additional links between groups, other than through the head node. It was further noted that none of the designers who used the system made use of the higraph facility.

Differences between users from a programming background and users from a graphic design background became apparent during the evaluation. Concepts in HANDIE such as class and list nodes, and the approach to data definition, required little explanation to the programmers, but were seen to be significant hurdles for graphic designers

\section{CONCLUSION}

This paper has described the development of HANDIE, a notation for the support of hypermedia document design. A prototype design environment based on this notation has been developed, and a preliminary evaluation carried out by several people involved in hypermedia document design. This experience both reinforces the idea of the general approach adopted, that of providing tools to support design rather than attempting to impose rigid methodologies, and suggests that there is definite merit in the simple yet flexible notation of HANDIE.

From the evaluation, it is possible to review HANDIE with respect to the four general principles mentioned earlier:

- Transparency: Nonprogrammers did experience some difficulties with concepts fundamental to HANDIE.

- Completeness: HANDIE was found to deal with most situations, but some additional abstractions were discovered to be desirable.

- Software Implementability: HANDIE has already been implemented as a computer-based design environment.

- Continuity of Support: HANDIE supports only part of the design process, but it is not inconsistent with what comes before and what follows on.

Obviously some further refinement of HANDIE should be carried out to bring it closer to these principles. Within the scope of the current prototype, the following refinements are currently being considered:

- A composite node facility should be developed, which will allow a single node to be composed of a number of elements, thus allowing for more than 
one list in a single node, or a node to combine a list with other information, for example.

- The list node concept should be generalised to allow conditional links from a list node to nodes other than class nodes, allowing lists of lists, or even lists of basic nodes.

- A mechanism should be provided to allow links from one design window to another, so allowing more flexibility in the use of group nodes.

- Consideration should be given to improving the methods for defining and representing higraph links.

- The interim mechanism for data definition should be reviewed.

Ultimately it is intended that HANDIE should be integrated with an appropriate data definition tool on one side, and move closer towards the generation of actual documents on the other. However, a production version of its present form would nevertheless provide valuable and usable support for the overall design process.

\section{REFERENCES}

Chen, P.P. (1976) The Entity Relationship Model. ACM Transactions on Database Systems, 1(1), 9-36.

Duncan, S. and Apperley, M. (1994) A graphical methodology for the design and implementation of hypertext based information systems, in OZCHI'94 Conference Proceedings (Melbourne, Australia, 28 November - 1 December, 1994) Ergonomics Society of Australia, Canberra, 161-166.

Ginige, A., Lowe, D.B. and Robertson, J. (1995) Hypermedia authoring. IEEE Multimedia, 2(4), 24-35.

Halasz, F. and Schwartz, M. (1994) The Dexter hypertext reference model. Comm. $A C M$, 37(2), 30-39.

Hardman, L., Bulterman, D.C.A. and van Rossum, G. (1994) The Amsterdam hypermedia model: Adding time and context to the Dexter model. Comm. $A C M, 37(2), 50-62$.

Harel, D. (1988) On visual formalisms. Comm. ACM, 31(5), 514-529.

Isakowitz, T., Stohr, E.A. and Balasubramanian, P. (1995) RMM: A methodology for structured hypermedia design. Comm. ACM, 38(8), 34-44.

Jul, S. and Furnas, G. (1997) Navigation in electronic worlds - a CHI 97 workshop. ACM SIGCHI Bulletin, 29(4), 44-49.

Maurer, H., Scherbakov, N. and Schneider, A. (1995) HM-Card: A new hypermedia authoring system. Multimedia Tools and Applications, 1(4), 305326.

Nanard, J. and Nanard, M. (1995) Hypertext design environments and the hypertext design process. Comm. ACM, 38(8), 49-56. 
Newcomb, S.R., Kipp, N.A. and Newcomb, V.T. (1991) The HyTime hypermedia/time-based document structuring language. Comm. $A C M, 34(11)$, 67-83.

Pohl, M. and Purgathofer, P. (1994) Problems of the development of a hypertext authoring tool, in OZCHI'94 Conference Proceedings (Melbourne, Australia, 28 November - 1 December, 1994) Ergonomics Society of Australia, Canberra, $167-172$.

Schwabe, D. and Rossi, G. (1995) The object-oriented hypermedia design model. Comm. ACM, 38(8), 45-46.

Schwabe, D., Rossi, G. and Barbosa, S.D.J. (1996) Systematic hypermedia application design with OOHDM, in Hypertext'96 Proceedings, The Seventh ACM Conference on Hypertext (Washington DC, March 16-20) ACM Press, $116-128$

Shih, T.K. and Davis, R.E. (1997) IMMPS: A multimedia presentation design system. IEEE Multimedia, 4(2), 67-78.

\section{BIOGRAPHY}

Mark Apperley is professor and chairperson of the Department of Computer Science at the University of Waikato, Hamilton, New Zealand. He has been actively involved in human-computer interaction research for the past twenty-five years. His current interests include information visualisation and presentation, computer-supported collaboration, and graphical design notations. 